\title{
HYBRID MODELS OF ALTERNATIVE CURRENT FILTER FOR HVDC
}

\author{
Ruslan A. Ufa ${ }^{1, *}$, Alexander S. Gusev ${ }^{1}$, Alexey S. Vasilev ${ }^{1}$, and Natalya G. Lozinova ${ }^{2}$ \\ ${ }^{1}$ Tomsk Polytechnic University, 634050 Tomsk, Russia \\ 2 JSC High Voltage Direct Current Power Transmission Research Institute, 194223, St.-Petersburg, \\ Russia
}

\begin{abstract}
Based on a hybrid simulation concept of HVDC, the developed hybrid AC filter models, providing the sufficiently full and adequate modeling of all single continuous spectrum of quasi-steady-state and transient processes in the filter, are presented. The obtained results suggest that usage of the hybrid simulation approach is carried out a methodically accurate with guaranteed instrumental error solution of differential equation systems of mathematical models of HVDC.
\end{abstract}

\section{Introduction}

The HVDC (high voltage direct current) systems are widely used in electric power system (EPS) with its technical and economic advantages [1,2]. However, operation specific of convertor stations of HVDC is the cause of harmonics which reduce the power quality and disturb the communication system [3,4]. Therefore, AC (alternative current) filters are necessary in convertor station to compensate harmonics, supply the reactive power consumed by the converter and mitigate current and voltage distortion in the EPS. AC filters, employing combination of resistors, capacitors and inductors, are used in three types of filter configuration - single tuned, double tuned and high-pass filters.

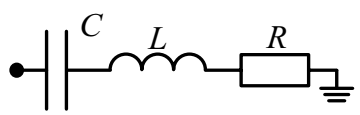

a)

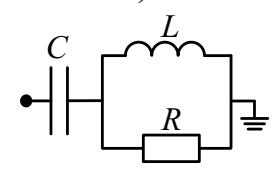

c)

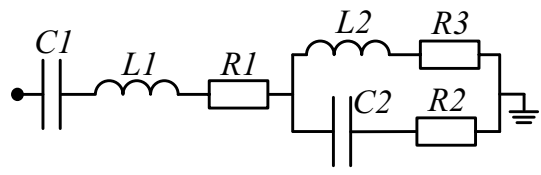

b)

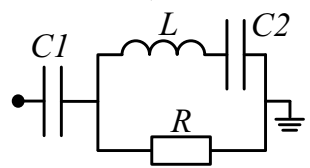

d)

Fig. 1. Configuration of AC filters: a) single tuned; b) double turned; c) second-order high pass; d) high-pass "C" type.

*Corresponding author: hecn@tpu.ru 
Single tuned AC filters is a series connected RCL circuit (fig. 1a) that are tuned at one harmonic frequency (in general, low order characteristic harmonics). Double tuned AC filters (fig.1b) are used to out two discrete frequencies (for example 5th and 7th or 11th and 13th harmonics). The main advantages of employing double tuned AC filters over the single tuned AC filters are: one reactor subjected to full line voltage, occupying less space, and needing to only one switchgear etc $[3,4]$. The damped filter or high-pass filter (fig.1c, d) offers low impedance over a broad band of frequencies. The high-pass filters are designed to damp more than one harmonic, for example, a filter tuned at 24th harmonic will give low impedance for both 23rd and 25th harmonic and for higher order harmonics. But the losses are higher than tuned filters.

In this paper the fragments of design of double turned and second-order high pass AC filter models for hybrid model of HVDC, presented in [2, 5], are introduced.

\section{Development of AC filter model}

According the basic principles of hybrid simulation concept for realization of HVDC model $[6,7]$ all power equipment of HVDC, which do not contain any switching elements, are described by mathematical models implemented via hybrid coprocessors. This approach is carried out the methodically accurate with guaranteed instrumental error solution of differential equation systems of mathematical models of HVDC by means of the continuous implicit integration method.

There are following steps for development of AC filter model: 1) formation and synthesis of hybrid mathematical model of the filter, providing a sufficiently full and adequate modeling of a single continuous spectrum of quasi-steady-state and transient processes in all normal, emergency and post-emergency conditions of filter operation; 2) usage of the continuous implicit integration method that carried out the methodically accurate with guaranteed instrumental error solution of differential equation systems of the model of filter; 3 ) the development of the specialized parallel digital-to-analog structures of the continuous implicit integration method, providing the methodically accurate solution of differential equation systems of the model of filter in real time and on an unlimited range.

\subsection{The double turned AC filter model}

The equivalent circuit and differential equation system of the double turned AC filter is presented in figure 2 .

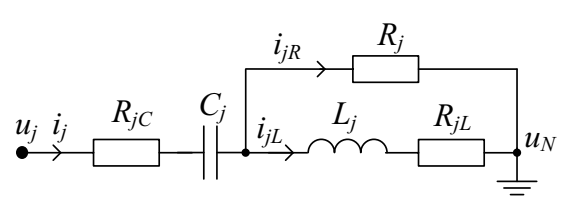

a)

$$
\left\{\begin{array}{l}
i_{j R}=\frac{1}{R_{j}} \cdot\left(u_{j}-i_{j} \cdot R_{j C}-u_{j C}-u_{N}\right) \\
\frac{d i_{j L}}{d t}=\frac{1}{L_{j}} \cdot\left(i_{j R} \cdot R_{j}-i_{j L} \cdot R_{j L}\right) \\
i_{j}=i_{j L}+i_{j R} \\
\frac{d u_{j C}}{d t}=\frac{1}{C_{j}} \cdot i_{j}
\end{array}\right.
$$

b)

Fig. 2. The equivalent circuit (a) and differential equation system (b) of the double turned AC filter, where $j=A, B, C$ - phase of filter; $R_{j C}$ and $C_{j}$ - active resistance and capacity of capacitor bank; $R_{j L}$ and $L_{j}$ - active resistance and leakage inductance; $R_{j}$ - resistor; $u_{N}$ - neutral voltage, $u_{j C}$ - voltage on reactive resistance of capacitor bank.

The developed specialized parallel digital-to-analog structure of the continuous implicit integration method, providing the methodically accurate solution of the differential 
equation system of the filter in real time and on an unlimited range and conversion of mathematical variable data input-output currents via $u / i$ converters in corresponding model physical variables are introduced:

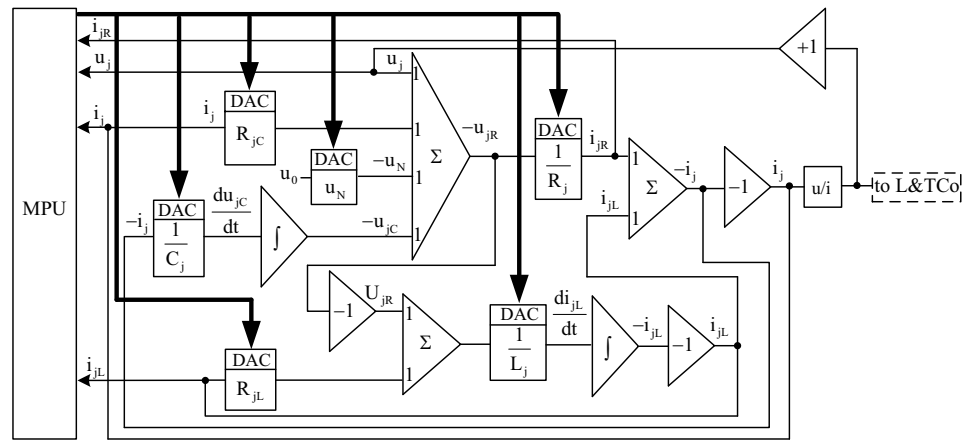

Fig. 3. The specialized parallel digital-to-analog structure of realization of the AC filter model, where all elements carrying out mathematical operations are based on integrated operational amplifiers in inverting gate, DAC - digital-analog converter, MPU - microprocessor unit, L\&TCo - longitudinaltransverse commutators, $u_{0}-$ reference voltage of DAC [2].

To confirm the adequacy of the developed model of the AC filter a frequency characteristics are obtained in Multisim 11.0 software and compared with the mathematical (base on eq.1) and theoretical frequency characteristics (based on the equivalent circuit of the double turned AC filter - figure $2 \mathrm{a}$ ):

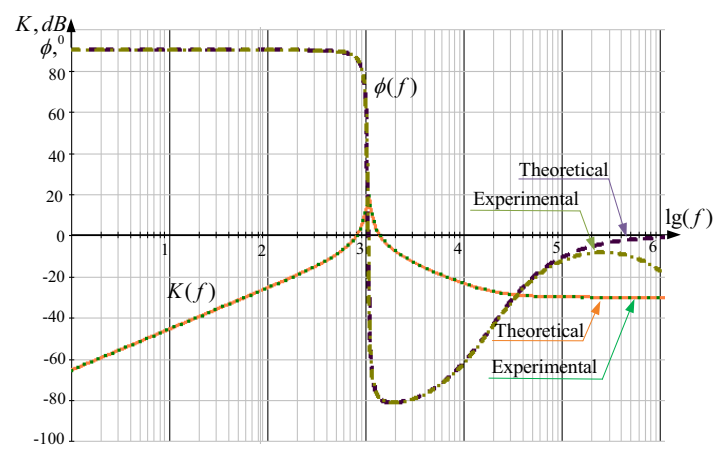

Fig. 4. Bode magnitude and phase plots of the double turned AC filter.

The obtained frequency characteristics confirm the correctness of filter tuning on the frequency $1050 \mathrm{kHz}$ (PWM switching frequency). The maximum value of the suppression ratio and phase reversal are observed at this frequency.

\subsection{The second-order high pass AC filter model}

The equivalent circuit of the AC filter tuned to suppress the 5th and 7th harmonics and analyze of model' frequency characteristic are presented in figure 5.

The obtained frequency characteristics confirm the correctness of filter tuning on the frequency $1050 \mathrm{kHz}$ (PWM switching frequency). The maximum value of the suppression ratio and phase reversal are observed at this frequency. The obtained frequency characteristics confirm the correctness of filter tuning on the suppressing the 5 th and 7 th harmonics. 


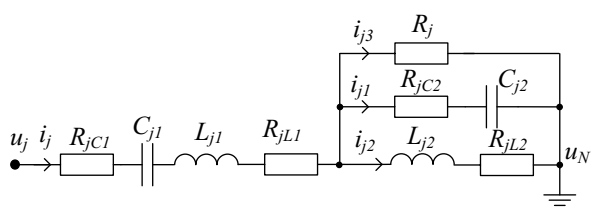

a)

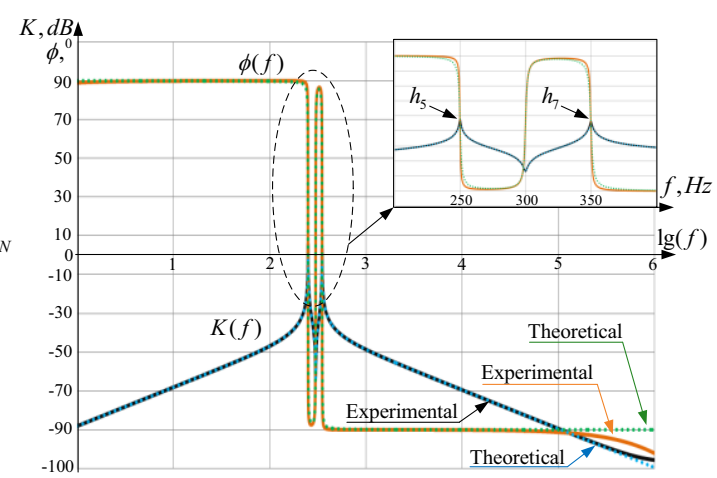

b)

Fig. 5. The equivalent circuit (a) and bode magnitude and phase plots (b) of the AC filter tuned to suppress the 5 th and 7 th harmonics.

Presented results suggest the following conclusions:

- the developed hybrid models of AC filter provide the sufficiently full and adequate modeling of all single continuous spectrum of quasi-steady-state and transient processes in all normal, emergency and post-emergency conditions of operation of the filter;

- usage of the hybrid simulation approach is carried out a methodically accurate with guaranteed instrumental error solution of differential equation systems of mathematical models of HVDC by means of the continuous implicit integration method.

The work was supported by Ministry of Education and Science of the Russian Federation under government grant «Science», project №3901: Research and development of hybrid model of back-to-back high voltage direct current transmission system.

\section{References}

1. M. I. Mazurov, A. V. Nikolaev, N. G. Lozinova, Improvement of voltage quality in AC network by use of STATCOM (IEEE Russia Power Tech, IEEE, 2005)

2. Yu. S. Borovikov, A. S. Gusev, A. O. Sulaymanov, R. A. Ufa, A. S. Vasilev, M. V. Andreev, N. Yu. Ruban, A. A. Suvorov, A Hybrid Simulation Model for VSC HVDC (IEEE Transactions on Smart Grid, IEEE, 2016)

3. M. A. Zamani, M. Mohseni, 9th International Conference on Electrical Power Quality and Utilisation, IEEE, 6, (2007)

4. L. Puming, H. Quanrui, Transmission and Distribution Conference and Exposition, IEEE, 6, (2008)

5. N. Yu. Ruban, A. S. Gusev, V. A. Sulaymanova, IOP Conf. Ser.: Mater. Sci. Eng., 93, $6,(2015)$

6. M. V. Andreev, A. O. Sulaymanov, A. A. Suvorov, MATEC Web of Conf., 37, 01001, (2015)

7. A. Suvorov, Y. Borovikov, A. Gusev, A. Sulaymanov, M. Andreev, N. Ruban, R. Ufa, Electrical Engineering (to be published) 